\title{
Video tracking and analysis of sleep in Drosophila melanogaster
}

\author{
Giorgio F. Gilestro
}

10

15
Department of Life Sciences, Imperial College, South Kensington, London SW7 2AZ, U.K., g.gilestro@imperial.ac.uk

Tel.: +44 2075945443 Fax: +44 2075947628

20

KEYWORDS: Drosophila melanogaster, actigraphy, video tracking, sleep, sleep deprivation, behaviour 


\section{ABSTRACT}

In the past decade, Drosophila has emerged as an ideal model organism to study the genetic components of sleep, its regulation and functions. In fruitflies, sleep can be conveniently estimated by measuring the locomotor activity of the animals using techniques and instruments adapted from the field of circadian behaviour. However, proper analysis of sleep requires degrees of spatial and

35 temporal resolution higher than is needed by circadian scientists, and different algorithms and software for data analysis. Here, I describe how to perform sleep experiments in flies using techniques and software (pySolo and pySolo-Video) created and developed in my laboratory. I focus on computer assisted video-tracking as instrument to monitor flies activity; I explain how to prepare the necessary equipment and how to plan a sleep analysis that covers the basic aspects of

40 sleep, closing with data analysis. A typical experiment for sleep analysis requires 5-7 days to perform.

\section{INTRODUCTION}

\section{Overview}

45 Drosophila melanogaster locomotor activity follows a tight circadian regulation: fruit flies are most active in the early hours of the morning and at dusk, and less active in between, with prolonged nearly continuous inactivity at night. This so-called crepuscular pattern of activity has been known since the early days - the very name Drosophila is Greek for "lover of the dew" - and the first systemic reports about the alternation of periods of activity and quiescence in adult Drosophila date

50 back to $1956^{1}$. However, despite a long standing interest in the circadian regulation of Drosophila activity, little was known about the biological characteristics of the mainly nocturnal resting state until the year 2000, when two independent laboratories demonstrated that the prolonged inactivity observed in Drosophila fully satisfies the five behavioural characteristics that define a sleep-like status $^{2}$ : (i) consolidated circadian periods of immobility, (ii) a species-specific posture and/or

55 resting place, (iii) an increased arousal threshold, (iv) reversibility to wakefulness and (v) a homeostatic regulatory mechanism ${ }^{3,4}$. We now know that sleep loss has similar detrimental effects on flies as it has on mammals ${ }^{5,6}$ and that social experience modulates sleep need in Drosophila, as it does in vertebrates ${ }^{7,8}$. Conservation of sleep from humans to flies extends beyond behaviour to pharmacology (e.g.: caffeine and amphetamines promote wakefulness ${ }^{3,4}$, whereas sedatives like

60 hydroxyzine promote sleep ${ }^{4}$ ) and flies, like vertebrates, show distinct electrophysiological correlates of wakefulness and sleep in the brain neuronal activity ${ }^{9}$. Together, these findings demonstrate an evolutionary conservation between Drosophila and human sleep at the behavioural and molecular levels, making flies an ideal system to study the mysterious functions of sleep and all its connections to pathological conditions in humans. 
65 Because sleep is associated with sustained physical inactivity, the easiest way to record sleep in flies is to measure their locomotor behaviour. Traditionally, this has been done using tools already employed to measure circadian rhythms, namely detecting when a single fly breaks an infrared light beam transecting the midline of a small recording chamber ${ }^{10}$ (Fig. 1a). Conceptually, this system dates back to the 1970s when Seymour Benzer adopted it in his mutagenesis screen for flies with

70 defective circadian rhythms ${ }^{11}$ and for the past 40 years this approach has indeed proved efficient for circadian research ${ }^{12}$. Infrared based monitoring of Drosophila locomotor activity does, however, carry drawbacks when applied to sleep: above all, low spatial resolution and the inability to record interacting flies. The former is particularly important since detecting movements only when the flies are crossing the midline has been reported to strongly overestimate sleep in some conditions ${ }^{13}$. Here

75 I recommend using an automated motion-tracking system based on computer vision analysis as it offers important advantages over traditional systems (see Table 1 and below). Automatic analysis of behaviour is an exciting emerging field in Drosophila neuroscience and several systems for computer assisted monitoring of sophisticated behaviours are currently available (Supplementary Table 1). In this protocol I concentrate on a system developed by our laboratory that was expressly

80 designed to be used for sleep detection and analysis, and carries specific features that other software with different aims do not provide. pySolo ${ }^{14}$ is a software for data analysis characterised by an easy to use, powerful interface; its current alternative would be to analyse and plot data using elaborate spreadsheets or scripts, such as Microsoft Excel macros and Mathworks MatLab scripts (Supplementary Table 1). pySolo-Video translates video-detected motion into activity data: it is

85 integrated with pySolo, it is highly scalable and relies on inexpensive hardware components.

\section{Comparing video tracking and infrared beam-split}

Differences between video tracking and the traditional system are summarised in Table 1. The main advantages of video tracking over infrared beam-split monitors are (i) a more faithful read out of

90 locomotor activity - and, consequently of sleep, (ii) very affordable costs, (iii) freedom in choosing the recording environment and (iv) the added value of the data recorded. The increase in spatial and temporal resolution of video tracking over the beam-split system is not due to an intrinsic weakness of the latter, but merely a consequence of the fact that traditional infrared monitors rely on only one beam per tube. In the single beam system, the infrared sensor intersects the midpoint of the walking

95 chamber and movements can be detected only if the fly is walking across (Fig. 1a). When the fly wanders on one side of the chamber, no movement is detected and sleep may be overestimated (Zimmerman et $\mathrm{al}^{13}$ and our unpublished data). To overcome this drawback, new monitors were recently commercialized that detect activity using three, nine and up to 16 infrared beams per tube (LAM and MB5, TriKinetics Inc). Data using these new high resolution monitors are not yet 
available in the scientific literature but it is easy to predict how an increase in spatial resolution will result in a more faithful read out. Video tracking, however, still offers a much higher level of resolution (an arena of 110 by $65 \mathrm{~mm}$ imaged at 640x480 pixels corresponds to a definition of less than $0.2 \mathrm{~mm}$ ). Difference in costs between the two systems will vary according to the number of monitors required and the level of resolution desired. A system of 30 video-tracking monitors will cost around $\$ 3000$, while the infrared split-beam alternative may range between $\$ 15000$ and $\$ 100000$, depending on the desired spatial resolution. Price difference is modest for a small installation but considerably more relevant for a bigger setup (Supplementary Table 2). However, the economic gain of adopting video tracking comes at the expense of time investment: commercially available infrared beam-split monitors (TriKinetics Inc.) were engineered to be ready

110 to use. The only assembly required is the connection of the recording monitors to the computer, which can be successfully done in few hours of work, or less ${ }^{15}$. The system for video tracking described here, on the other hand, requires the experimenter to assemble, test and adapt all the hardware equipment, an effort that may require days depending on the experience and ability of the experimenter. A final important advantage of video tracking over infrared beam-split monitors is the

115 ability to host and record flies in an environment different from the traditional glass cuvette. Due to engineering design, beam-split recording must be performed in sealed vials of limited size (Fig. 1a); video tracking, on the other hand, can detect movement independently of the size and complexity of the environment in which the fly is located, allowing the researcher to adopt arena that are more convenient to use - like the ones described in this protocol - or suitable to complex experiments.

120 For instance, video tracking is commonly used to monitor flies interacting with each other in an open environment and it is technically possible to detect activity and sleep of several flies walking freely in a Petri dish. However, to unequivocally resolve the identity of single flies over prolonged times is still an open problem of computer vision and this kind of experiment should either be treated as "population readings" (rather than "individuals readings") or else be performed by

125 marking flies beforehand, using paints of different colours. Finally, video tracking offers an added value to the kind of data recorded: by analysing the Cartesian coordinates of a fly at any given time, the researcher can obtain information on a number of kinematic aspects such as speed, directions, preference in local positions.

\section{Experimental workflow}

A typical sleep experiment consists of three phases: a preparative phase; a recording phase; and a data analysis phase (Fig. 2). In the preparative phase, flies are prepared for the experiment, with stocks or crosses expanded as required. At the appropriate age, flies are transferred to the arena that will host the animals throughout the recording and provide them with the necessary nutrients. 
135 Recording typically lasts 5-7 days and requires little or no human intervention. Flies are first monitored in a controlled environment for two or three consecutive days, to score their baseline sleep needs; sleep deprivation can then be performed, followed by a third phase of recovery sleep to study rebound. Data collected during the recording phase can be analysed at any time and from any computer.

140

\section{Experimental design}

Defining sleep. Flies that are sleeping are not moving, but flies that are not moving are not necessarily asleep. The best way to distinguish locomotor rest from sleep is to measure arousal threshold. When flies are asleep, their arousal threshold increases and it is therefore possible to 145 reliably infer if an animal is asleep by actively challenging it with a sensory stimulus, such as tapping $^{3,16}$ or vibrating ${ }^{4}$ the vial. The likelihood that a fly will respond to a complex stimulus levels off after five minutes of immobility ${ }^{16}$ and for this reason sleep in flies is commonly defined as "a period of inactivity lasting at least five consecutive minutes". This definition implies that our estimate of sleep is a linear mathematical derivation of the measure of activity and explains why a more careful reading of activity will result in a more faithful estimate of sleep (Fig. 3, panels e,f. See Box 1 for a glossary providing definitions for other aspects of sleep and sleep monitoring).

Choice of computer. In ideal conditions, the experimenter will have access to at least two computers: one dedicated to video tracking and located in the laboratory where the experiments are

155 taking place, and one for data analysis. The video tracking part of the software is written in Python using the Open Source Computer Vision Library (opencv, Intel Inc.) and will run on any major operating system (Ubuntu Linux 10.10 is the recommended choice). A consumer business desktop PC, such as HP Compaq 8200 or Dell Vostro 460, will handle 30 video monitors and possibly more (we have not tested our system with more than 30 monitors). There are no special requirements for 160 the computer used to analyse data.

Choice of the video camera. A setup for video analysis relies on four main components: a video camera; a recording arena where flies are housed; software to collect images and analyse movements; and software to analyse data (Fig. 1b). Virtually any video camera that can be 165 connected to a computer will work with pySolo-Video given that there is no need for high refresh rate or high resolution. The only requirement is the ability to capture images under infrared light conditions during the dark hours. We routinely use inexpensive cameras normally commercialized for video conferencing (webcams, see MATERIALS): for less than $\$ 5$ they offer enough image resolution (640x480 pixels), more than sufficient refresh rate $(\sim 10-20$ frames per second) and the 
ability to record under infrared illumination. A single camera can record over 30 flies simultaneously from a distance of about $20 \mathrm{~cm}$. Figure $3 \mathbf{a}$ offers a representative snapshot of a video collected using a similar camera.

The recording arena. In experiments measuring circadian rhythms, flies were traditionally housed 175 in small glass cuvettes, containing fly food on one end and cotton wool on the other (Fig. 1a and Supplementary Figure 1b). However, video analysis offers added freedoms in the choice of recording arena ${ }^{17}$. We use and recommend custom designed plates obtained through $3 \mathrm{D}$ printing techniques (Fig. 1b and Supplementary Figure 1b,c) because they provide more consistency between samples, are inexpensive and, most importantly, their preparation requires less time

180 (minutes versus hours or days). Custom recording arenas can be virtually modelled using 3D modelling software and then printed in house or using third party printing services. Alternatively to 3D printed models, the experimenter still has the choice to use traditional glass tubes or multi-well plastic containers normally adopted for cell cultures (see EQUIPMENT).

185 Illumination. Flies must be illuminated using two different sources of light: visible light during the subjective day, and infrared light during the dark hours. In both cases, direct illumination of the recording arena may result in reflective glare affecting the quality of the image acquisition, a particularly frequent issue when flies are illuminated by an infrared light located above the arena, for instance when using the LEDs integrated in the camera itself. For best results, disable or remove 190 the LEDs in the camera and place an independent infrared LED strip underneath the arena, illuminating the flies from below: this will minimise the glare and increase contrast (see Fig. 3a for a snapshot of a nocturnal video taken using back illumination).

For both visible and infrared illumination, we recommend using low voltage LED strips as they are easy to wire and install and provide controlled intensity of light without sensibly raising the 195 temperature in the recording chamber (see MATERIALS).

Planning the experiment. A typical experiment may be composed of three successive phases (Fig. 2): baseline sleep (2-3 days), Fig 2a; sleep deprivation (6-24hours), Fig 2b; and recovery sleep (2-3 days), Fig 2c. During baseline sleep flies are left undisturbed and each individual animal is monitored to set their baseline sleep need. On the third or fourth day, flies may undergo sleep deprivation using a protocol of choice (see 'Interfering with sleep', below). Homoeostatic response to sleep deprivation should manifest in the days following sleep deprivation, during which flies are tested for their ability to recover sleep loss. 
205 Interfering with sleep. The ability to interfere with sleep is as important as measuring sleep itself. Rebound following sleep deprivation is a hallmark of sleep, and sleep deprivation has important consequences on a multitude of conditions. Flies, like vertebrates, show both cognitive and physical impairments upon prolonged sleep deprivation with consequences that range from learning deficits to death ${ }^{6,18}$. There are several ways to perform sleep deprivation in Drosophila: pharmacological; 210 mechanical; social; and starvation-induced. Table 2 provides a summary of strengths and weaknesses of each of them but in the text procedural details are only provided for mechanical and pharmacological manipulation of sleep as they are most relevant to a broader audience

- Pharmacological manipulation of sleep. Many pharmacological compounds that modulate sleep in vertebrates act on insects too (reviewed in Table 3). Sleep or sleep loss can be

215 induced by feeding flies with food containing a sleep-altering compound. It is important to keep in mind that drugs may have side effects which extend beyond sleep control and complicate the readout of the experiment; however, there are cases when the experimenter may want to assay the response of a given genotype to specific compounds, for instance to test whether a gene acts in a specific sleep regulating pathway.

220 - Mechanical disruption of sleep. It is possible to obtain robust sleep deprivation through mechanical stimulation of flies: for instance, tapping the vials where the flies are housed whenever they stop moving ${ }^{3}$, spinning the flies upside down to interfere with their geotactic instincts $^{6,18}$, or placing flies on a laboratory orbital rotator ${ }^{19}$. Manual sleep deprivation is efficient but incompatible with high throughput experimental conditions; automatic sleep

225 deprivation provides a good compromise and can be achieved by placing the monitor in a rotating device, which is relatively easy to build ${ }^{18}$, or on a common laboratory orbital shaker $^{19}$

- Socially-induced suppression of sleep. Sleep loss occurs when a socially naïve experimental fly is suddenly disturbed by another introduced into the arena. Socially230 induced sleep suppression may have greater ecological relevance than mechanically induced sleep suppression, but will work only when using male-male coupling (reference 7 and unpublished data, G.F.G.); other kinds of interactions may still induce changes in sleep behaviour that may be interesting to study ${ }^{8}$. Locomotor activity during social interaction must be measured using video tracking techniques; however, completely automatic tracking 235 of multiple flies interacting in an open arena for prolonged times is still difficult for computers and may require significant corrections by a human observer ${ }^{20,21}$.

- Starvation-induced sleep suppression. This behaviour is conserved across phyla and may be particularly important given that sleep-feeding interactions have been linked to diseases including obesity, diabetes and metabolic disorder. Switching the food source from standard 
fly food to agar, where they attain their water needs with no nutritional benefit, results in robust suppression of sleep ${ }^{17,23,24}$.

Controls. Experimental groups should be carefully matched to reduce any influence of uncontrolled factors, such as the genetic background of the animal. A good description of this problem and how 245 to address it were discussed by Rosato and Kyriacou ${ }^{12}$ and I refer to them for more details. In particular, male and female flies show radically different sleep patterns, with males undertaking a significantly longer post-meridian nap (also called $\operatorname{siesta}^{24}$ ) than females. Sleep patterns also change with age $^{25}$, social experience ${ }^{8}$ and feeding status ${ }^{17,23,24}$. Therefore it is critical to compare flies of the same sex, age and with similar previous experience. Genotype should also be considered and comparisons within Drosophila stocks with dissimilar genetic background should always be avoided. When comparing flies expressing transgenes under control of the GAL4-UAS system, it is advisable to start by crossing heterozygous parental stocks, so that the progeny will be composed of experimental animals and their sibling controls, distinguishable by eye colour or, post hoc, by PCR genotyping.

255 Sample size should vary as a function of statistical power, that is the difference in the mean values between experimental and control group and the internal variability within each group. Many sleep parameters (e.g.: sleep duration) tend to be relatively homogeneous within an isogenic population; others (e.g.: sleep deprivation efficiency) will vary greatly even within sibling flies. Therefore, sample size should be empirically determined based on the experimental conditions and may vary from as little as 16 flies per group to a few hundred ${ }^{12,26}$.

Environmental conditions. Temperature, humidity and light intensity should be kept constant with time and within groups. Any external stimulus, including noise, should be avoided and experiments should run in a dedicated environment, preferably in dedicated incubators.

\section{MATERIALS}

\section{REAGENTS}

A Fly food: The Bloomington Drosophila Stock Centre provides methods and recipes for cooking different kinds of fly media (http://flystocks.bio.indiana.edu/Fly Work/media$270 \quad$ recipes/media-recipes.htm ).

A Organic cotton wool (Genesee Scientific, cat. no. 52-101).

\section{EQUIPMENT}


A Vials and plugs for raising flies (plastic flat bottom tubes $75 \times 23.5 \mathrm{~mm}$, Regina industries, cat. no. P1014L, Regina industries, cat. no. 49-102)

A Narcotic pad (Genesee Scientific, cat. no. 59-114)

A Fine artist's paintbrushes for handling flies.

A A computer for recording data. Computer specifications will vary depending on the number of cameras recording simultaneously in the system. For up to four cameras, a "deskbook like" computer will suffice (e.g.: Acer Aspire Revo, Atom D525 1.8GHz processor, 2GB ram, $250 \mathrm{~Gb}$ disk). A more powerful system will handle many more cameras (e.g.: HP Compaq 8200 , Intel i7 $2.8 \mathrm{GHz}$ processor, $4 \mathrm{~Gb}$ ram, $500 \mathrm{~Gb}$ Disk). Number of USB ports is not critical as they can be easily expanded using USB hubs (see below). The computer should be connected to the network for data accessibility.

A Recording and analysis software (http://www.pysolo.net)

A One or more video cameras with USB connection CRITICAL The camera must be able to see under infrared (IR) light conditions. Many cameras carry an IR filter that would have to be forcefully removed. Cameras that provide in-built IR-LEDs do not have such a filter and are therefore preferred (we routinely use http://www.ebay.com for inexpensive $(<\$ 5)$ cameras with integrated IR LEDs such as Kinamax WCM-6LNV).

A One or more USB hubs (D-Link DUB-H7) CRITICAL The hub should be powered by an external power supply or cameras may not receive enough current to work properly

A Visible LED lights ( LEDLighting.co.uk, cat. no. 154798)

295 A Infrared LED lights (LEDlightsworld.com, 850nm Tri-Chip LED). Both visible and infrared LED strips are sold by $5 \mathrm{~m}$ reel but can be conveniently cut to fragments of $5 \mathrm{~cm}$ and wired directly to a power supply.

A A digital mains timer socket (Amazon.co.uk, cat. no. B000TBGWIO)

A Adhesive magnetic strips (RS components, cat. no. 297-9093)

300 A Food grade plastic container (Lock \& Lock Storage 1.6L Medium Container)

A One or more recording arenas to host flies. A purposely designed arena can be custom made using 3D printing (see EQUIPMENT SETUP)

A PETG Clear copolyester sheet (RS Components, cat. no. 334-6444)

A Multi-well cell culture plates (BD Falcon, cat. no. 353503) or glass tubes (Trikinetics, 305 Waltham, MA). These are ready to use alternatives to the 3D printed arena.

A Optional: a Drosophila incubator (Percival Scientific)

A Optional: a sleep deprivator. A rotation device can be custom built or can be adapted from a laboratory orbital rotator (Cute Mixer CM-1000, EYELA or VWR VX2500 with a TriKinetics adaptor) 


\section{REAGENT SETUP}

Fly maintenance Grow flies in vials, keeping them in constant conditions of light, temperature and humidity. An artificial light-dark (LD) regimen of 12:12 is considered standard; temperature should be constant, between $21^{\circ} \mathrm{C}$ and $25^{\circ} \mathrm{C}$; humidity should be kept above $60 \%$. Avoid overcrowding

315 by removing adult parent flies 3 to 5 days after setting up the vial.

Preparing food for the arenas Place a vial with fresh food in the microwave oven and heat for 10 seconds or until melted. Stir food with a pipette to remove clumps and heat few more seconds. For experiments using drug-induced sleep suppression, substitute fly food with a $1 \%$ agar water solution or food laced with the desired drug.

\section{EQUIPMENT SETUP}

Computer and software Place a computer next to the incubator where experiments will run. Any major operating systems will work, including Microsoft Windows and MacOSX, but for a brand new installation we recommend using Linux Ubuntu, version 10.10 (http://www.ubuntu.com).

To Install pySolo Video on Windows or Linux, point your internet browser to the pySolo website (http://www.pysolo.net), go to the Download section and select the latest packaged version. Double click on the file to install it. On Ubuntu Linux, subscribe to the pySolo repositories to have automatic updates of new releases. Supplementary Video 1 offers step-by-step instruction on how

330 to install pySolo and pySolo-Video on Linux Ubuntu 10.10.

Print the recording arena The arenas described in this protocol are obtained using 3D printing technology. We use an Eden 250 (Objet) using VeroWhite FullCure830 as printing material. With our printer, the cost of printing an arena like the one shown in Supplementary Fig. 1b is around \$20. STL file ready to print is provided as supplementary data and can be open and visualised using

335 the free software meshlab (http://meshlab.sourceforge.net). Many third parties services offer remote 3D printing for comparable prices (e.g.: http://www.shapeways.com). Whether printed in house or by third parties, we recommend stereolithography, as the final product will be smoother and easy to clean under running tap water.

Prepare the lids for the recording arena Cut the copolyester sheet to fit as a sliding cover for the arena (63mm x 100mm, Fig. 1b and Supplementary Figure 1b). Cut a larger ( $>80 \mathrm{~mm}$ x $100 \mathrm{~mm})$ portion of the copolyester to be used as a transferring lid. The transferring lid should be wider than the normal lid and have two holes of about $1 \mathrm{~mm}$ diameter through which flies will be blown in (Fig 1b).

Build a recording box To build a recording box drill a hole through the bottom side of the food 
345 container (i.e.: opposite to the lid) and place the camera so that the lens will look through the hole. Use glue or screws to secure the camera to the surface so that it will not move even when the container is shaken. Position the recording arena with flies on the internal side of the lid. Use self adhesive magnetic strips on both sides to fasten the arena to the inner surface of the lid. Cut a small strip $(5 \mathrm{~cm})$ of visible LED lights and place it inside the container to illuminate the flies during

350 subjective day. Regulate timing of light transitions using a digital timer connected to the power supply. See Figure 1c for a schematic representation of the recording box and Supplementary Video 2 for a detailed description of how a recording box is built.

Connect the camera to the computer through the USB port, using an intermediate USB hub if necessary. Place the recording box in an environment with controlled light, temperature and

355 humidity (i.e.: ideally an incubator or, if not available, an undisturbed cabinet in the fly room).

\section{PROCEDURE}

\section{Preparing the experimental animals TIMING 13 days at $25^{\circ} \mathrm{C}$}

1) Approximately two weeks prior to the planned start of the experiment, prepare one or more vials with the lines to be analysed and compared.

2| Allow flies to mate and lay eggs for 3 to 5 days then remove parents and wait for progeny to develop to imago.

\section{Preparing activity arena TIMING 20-30 minute}

3| Open the arena by sliding the transparent lid and fit the separation walls around the food chamber to avoid spilling.

CRITICAL STEP See Supplementary Video 3 for a step-by-step description of how to prepare and load an arena.

4| Prepare food to be transferred to the arena (see REAGENTS SETUP).

3705 Using a syringe, transfer melted food to the inner chamber of the arena and then wait for it to solidify (about 10 minutes at $25^{\circ} \mathrm{C}$ ).

6| Remove the separation walls, place some cotton wool on the external edges of the arena and slide the lid in from the side.

375 PAUSE POINT Multiple arena can be prepared at once and then stored a $4-10^{\circ} \mathrm{C}$ for a few days.

\section{Loading the activity arenas TIMING 20 minutes}

7| Collect flies 1 or 2 days after pupal eclosion and place them in new vials in small groups of $\sim 10$ individuals of the same genotype, to avoid overcrowding and to make sure that all the 
flies are of the same age. If flies must be sorted to separate different genotypes, you may need to use $\mathrm{CO}_{2}$ anaesthesia on a narcotic pad.

CRITICAL STEP Compare flies of the same sex and age and make sure flies are of normal size with fully expanded wings. Sick flies will show strong differences in sleep/wake regulation.

CRITICAL STEP If using females, make sure to collect virgins; mated female flies will lay eggs and larval development in the recording environment should be avoided because crawling larvae will interfere with motion detection and consume the food.

\section{TROUBLESHOOTING}

$8 \mid$ When flies are at the correct age (normally 5 days old), transfer them to the arena. Flies can be transferred to the arena using two systems: transferring sedated animals (using $\mathrm{CO}_{2}$ or cold) (option A) or transferring the awake animals using a mouth-pipette (option B).

Supplementary Video 3 contains a detailed explanation of both procedures.

\section{TROUBLESHOOTING}

\section{(A) Transferring flies using $\mathrm{CO}_{2}$ sedation}

(i) Sedate flies using a narcotic pad connected to $100 \% \mathrm{CO}_{2}$.

(ii) Using a small paintbrush, transfer each flys from the pad to a single groove in the arena (iii)Close the lid by sliding the clear plastic in

\section{TROUBLESHOOTING}

\section{(B) Transferring flies using a mouth pipette (fly sucker)}

(i) Slide the transferring lid into the arena, so that the portion with the two holes covers the first groove

(ii) Insert the tip of the mouth-pipette into the vial containing the flies, through an opening in the cotton wool stopper

(iii)Suck a single animal into the tip of the pipette

(iv)Transfer the animal into the arena by blowing it through the hole in the transferring lid; transfer one fly to each side of the arena

(v) Gently slide the transferring lid to cover the next grooves in the arena

(vi)Repeat from $8 \mathrm{~B}$ (ii) until all the required flies have been transferred.

(vii) Replace the lid with another of the correct size that has no holes in it

TROUBLESHOOTING

CRITICAL STEP When comparing experimental and control animals, it is always preferable to host both in separate grooves in the same arena, mixing 16 control flies to 16 experimental animals, either randomly or placing 16 flies on each side of the arena. This will reduce the operational variability. 
415 Remember to keep a record of fly genotypes and location in the arena

9| Place the arena with flies in the recording box and place the box in the incubator.

CRITICAL STEP Let the animals recover and habituate for at least one night before starting any experimental recording, especially if $\mathrm{CO}_{2}$ sedation was used to load the arena. Social experience does have an effect on sleep amounts and regulation so allow one or two extra days of habituation if flies loaded in the arena were previously kept in a socially enriched environment.

\section{Preparing the pySolo software for data fetching TIMING 30 minutes}

CRITICAL STEP The steps from 10 to 13 are configuration steps, required only once at the

\section{5}

Pr

beginning of the procedure or whenever the location where they are saved changes

10| Supplementary Video 4 provides a step-by-step guide on how to set up automatic formatting and collection of data using the datafetcher component of pySolo. Briefly, navigate to the directory where the datafetcher is installed.

11| Using your favourite text editor open the configuration file copyfiles.cfg and change the relevant parameters. An explanation of each variable is offered in the Supplementary Video 4: inputpath is the path of the rawdata; outputpath is the path to the processed data that will be used by pySolo for analysis; zippath is the path where a backup copy should be saved. For the latter, it is recommended to use an external hard drive or a network disk for sake of data safety.

12| Add the script copyfiles.py to your scheduled tasks so that it will run daily, moving the data into a directory tree organized by date and monitor numbers.

\section{Preparing the pySolo-Video software for data recording TIMING 1 hour}

13| Supplementary Video 5 provides step-by-step instructions on how to get started with pySolo-Video. Briefly, the first time you start the program, open the option panel to customize the total number of available webcams and monitors, as well as the path where rawdata will be saved (same as inputpath in step 11).

$14 \mid$ Restart the program to reload the new settings.

15| At the beginning of each experiment, associate each camera to its own monitor by clicking on the thumbnail in the thumbnail panel and selecting the appropriate source from the dropdown menu found in the "Select Video Input" box.TROUBLESHOOTING 
16| Move to the live view panel in the program interface.

17| Using the mouse, draw a mask to delineate and separate the area in which a single fly will move.

18| To create a mask either drag the cursor on the screen holding down the left button of your mouse, or click on four arbitrary corners to draw a polygonal irregular area. Click on the middle mouse button to confirm the selection and activate the mask. The first mask you draw will be "channel one" of your monitor, the second mask will be channel two and so on. The live view panel will show real time analysis of flies activity as you draw to mask (Fig 3a).

CRITICAL STEP Each mask should cover only one groove or channel. Pay special care in drawing accurate borders. If you make any mistake, select the mask by clicking inside it and delete it clicking the right mouse button

19| Save the mask with a chosen name.

$20 \mid$ Go back to the thumbnail panel

21| For each monitor, decide the format to save the data (see Fig. 3b-d for an explanation of the different possibilities) and associate the mask you just created, then hit the Apply button

22| Save all your settings in a configuration file

23| Close the pySolo-Video-GUI

\section{Recording data TIMING 1 week}

$470 \quad 24 \mid$ Start the pySolo-Video-Acquire component.

25| Click on Browse and locate the configuration file you saved on step 22. Hit Open.

26| The Acquire window will show a table with a list of all monitors previously configured

27| Hit Start to begin tracking

\section{Interfering with sleep}

28| Advantages and characteristics of the different methods to alter sleep are discussed in the Experimental Design section. Here follows a more detailed description of the commonly used kinds of sleep interference: option (A) Mechanical disturbance and option (B) Pharmacological modulation.

\section{TROUBLESHOOTING}

\section{(A) Mechanically induced sleep deprivation TIMING 3-24 hours}

(i) Record baseline sleep for 48-72 hours 
(ii) The day the disruption is due to start, place the recording chamber on the rotating device.

(iii) Switch the orbital rotator on.

CRITICAL STEP High rotation speed, especially if performed for long stretches of time $(>3 \mathrm{hr}$ ) may be damaging and kill the flies. Optimal speed settings may vary depending on the orbital shaker and should be empirically calibrated. Start at very low speed and go higher if necessary. A sleep deprivation of six hours is normally sufficient to induce noticeable effects. Sleep deprivations that extend beyond the 24 hours may result in death. Sick stocks may die even earlier ${ }^{19}$

(iv) After the required time has elapsed, end the sleep deprivation and place flies back in the incubator.

\section{TROUBLESHOOTING}

\section{(B) Pharmacologically induced sleep deprivation TIMING 12-48 hours}

Pharmacological sleep deprivation can be achieved by manually transferring flies from an arena with control food to another with drug-enriched food.

500 (i) Prepare two arena: one filled with control food and one with laced food (either 1\% agar or food with chemicals for pharmacological manipulation. Table 3 provides a review of all pharmacological manipulations shown to have an effect on Drosophila sleep).

(ii) Measure baseline sleep keeping flies in the control food for 48-72 hours.

(iii) At the beginning of the manipulation time, use a mouth-pipette to move flies from the 505 arena with regular food to the one with laced food. Place the arena back in the recording chamber.

(iv) Record treated flies for 24 hours then, transfer flies back to the arena with untreated food using a mouth pipette as described in step 28B (iii).

510 CAUTION Sleep is an evolutionary conserved phenomenon and some drugs may have neurological effects on humans as on flies. Handle with care.

\section{TROUBLESHOOTING}

29| Measure rebound following sleep deprivation for at least 48 hours.

\section{Cleaning the arena and discarding the flies TIMING 10 minutes}

$30 \mid$ At the end of the experiment, remove the arena from the recording box. 
31| Fill a container with warm water and place the arena inside. Open the lid while still in the water and use a small brush to remove flies, food and cotton wool

$32 \mid$ Gently brush the arena under warm tap water, then rinse in deionised laboratory water and let dry.

33| Optionally, the arena can be washed using 70\% ethanol or, depending on the material adopted for printing, placed in a dish washer or autoclaved.

CAUTION Wash the arena soon after each use. With time, food will dry inside and will be more difficult to clean.

\section{TROUBLESHOOTING}

\section{Preparing the pySolo software for data analysis TIMING 30 minutes}

34| Supplementary Video 6 and supplementary manual provide a detailed description of how to configure and use pySolo for data analysis. Start installing pySolo on your office computer or laptop.

$35 \mid$ The first time you start the program you will be asked to provide some basic parameters in 535 the options panel. This configuration step is only required once, the first time the program is started. You will have access to the option panel at any given time later on, but three options must be specified immediately for pySolo to work: the path where the program will find the daily data (same as outputpath from step 11); the extension of the files containing the daily data (txt by default); the format of the input data (see also Fig. 3B-d). pySolo must have access to the folder where the daily are located to work

\section{Analysing data using pySolo TIMING 30 minutes-2 hours}

$54536 \mid$ In the database window of the program, load your experimental database file or create a new one.

37| Hit the button with a green plus sign to add new rows to your database

38| Fill in data about your experiment in each row: starting monitor, starting channel, end monitor, end channel, genotype, comment and the days of the experiment. See also the documentation on the pySolo website for more information (http://www.pysolo.net/)

39| Save your file

$40 \mid$ Select the lines of the experiments you want to analyse by marking their corresponding check box on the left side of the table

41| Point your mouse to the menu bar and click on Analysis > Check Raw Data files to make 
sure all the data the programs need are properly accessible and formatted

42| Click on Analysis > Fetch Raw Data to fetch the data

$43 \mid$ Save the resulting file with extension ".dad"

PAUSE POINT The resulting dad file can be opened into the analysis window of pySolo at any later time. Analysis can also be performed on a different machine since it is now completely independent by the original raw data.

44| Send the acquire data to the analysis window by either clicking on Analysis > Send Data to Analysis or opening the analysis window from Windows submenu of the menu bar and dragging and dropping the $\mathrm{dad}$ file inside.

45| Click on the Browser tab to open the Browser panel

46| Use the navigation tree on the left to select single genotypes, single days or single animals. Use the Control key on your keyboard to join together values from multiple selections

47| When comparing different experimental groups, use the button marked with a blue plus sign on the bottom left hand side to add new item to the analysis.

48| Evaluate and compare values of the most important aspects of sleep (sleep duration, rebound to sleep deprivation etc. See Fig. 4 and the Glossary for other examples). The software will automatically look for dead flies, whose sleep pattern is beyond predetermined threshold values. By default, flies sleeping longer than 1350 minutes a day are considered dead. If necessary, thresholds levels can be modified in the options panel.

49| If necessary, export numerical data by clicking on the table to be exported with the right mouse button. Files will be saved in a spreadsheet compatible format to allow external analysis or statistical comparison

50| Similarly, export graphical data as images using the right mouse button

\section{TROUBLESHOOTING}

580 Troubleshooting advice is provided in Table 4.

\section{TIMING}

Steps 1-2, preparing the animals: 13 days. Plan your crosses or expand your stocks in advance of the experiment so that enough flies will have hatched by the beginning of the experiment. For weak or sick stocks it is wise to setup more than one vial

Steps 3-6, preparing the arena: 20-30 minutes. Multiple arena can be prepared at once: add 1 more minute per each additional arena

Steps 7-9, loading the arena with the animals: 20 minutes per arena. Transferring awake flies in the arena using a mouth-pipette requires some experience and may be frustrating the first times. Give 
590 yourself up to one hour if you are new to this procedure and use some time to practice Steps 10-12, preparing the software for data fetching: 30 minutes (needs to be done only once).

Steps 13-23, preparing the software for data recording: 30 minutes.

Steps 24-28, recording activity data: about 1 week. See also Fig. 2 for a better break down of experimental times during data recording.

595 Steps 29-32, cleaning the arena: less than one hour. Preparing and cleaning the arena takes considerably less time than working with regular glass tubes (compare reference 14 to Supplementary Movie 3)

Steps 33-34, preparing the software for data analysis: 30 minutes (needs to be done only once).

Steps 35-49, analysing the data: 0.5 - 2 hours. Data analysis can be performed at any time and from

600 any computer on which pySolo is installed.

\section{ANTICIPATED RESULTS}

\section{Quality of image acquisition:}

Best results require optimal conditions of image acquisition by the camera. A successful setting

605 should result in an image whose quality is akin to the image in Figure 3a. Flies are detected with good contrast against the background so that even wings and legs are recognizable despite the low resolution of the camera; no glare should be observable or should be limited to areas that are not included in the recording mask.

\section{Internal controls:}

Duration of sleep and response to sleep deprivation will change considerably from line to line, even within wild type stocks ${ }^{17}$ whereas pattern of activity throughout the day should remain roughly comparable among control stocks. A good setup should show a robust difference in activity between the relative day and relative night and an appreciable difference in the daily sleep pattern when

615 comparing male to female flies. Peaks of activity should clearly appear at dusk and dawn. For these reasons, I recommend starting with pilot experiments to test the setup using wild type (Canton S or Oregon R) male and female flies. Take Figure 4a as an example of a good control experiment in which male and female sleep show a clear difference between night and day and where the male siesta is clearly visible.

620

\section{Acknowledgements}

I am deeply indebted to Alex Keene for his help with a first version of this manuscript. I would like to thank the open source community for creating and sharing valuable software. 
625 Competing financial interests

The author declares that he has no competing financial interests. 


\section{Figures legend.}

\section{Figure 1: Principles of locomotor detection}

630 A. Infrared beam-split measurement. A single fly is hosted in a glass tube, secured between food and cotton. A beam of infrared light crosses the midline of the tube (dashed red line) and a collector connected to a computer can record and count whenever the fly is breaking the beam by passing through it. If the fly moves away from the beam or if the fly sits right in the middle of it, too little or too much movement may be detected.

635 B. Arena for video recording. Arenas of different shapes and complexities can be produced using 3D printing technology. The cartoon depicted in this figure shows an arena hosting up to 32 flies, housed in parallel walking grooves covered with a lid of transparent plastic. On one end, flies are in contact with cotton and on the other end with food. Flies can be transferred to the arena either sedated or unsedated; in the latter case, a mouth pipette is used to blow single animals through holes in a transferring lid (blue circles in the figure). A STL file ready to be used for 3D printing is provided as Supplementary Material (see MATERIALS).

C. An expanded view of a video recording chamber. A food grade plastic container can conveniently house an arena similar to the one depicted in panel B. The arena is secured to the inner side of the lid through a paired couple of adhesive magnetic strips. A sheet of clear plastic slides onto the top of

645 the arena and keeps the flies from escaping. The camera looks at the flies through a hole in the opposite side of the container. Lighting can be provided either externally or with LEDs placed inside the container (not shown). A chamber built in this way will record flies in a closed environment and will resist to shaking and manipulations.

\section{Figure 2: Experimental workflow}

The three main phases of the sleep assay. During the preparative phase (A), flies of the correct age are inserted into a monitoring arena previously loaded with food. Typical recording (B) may last for four to seven days. At the end of the recoding, flies are discarded, the arena cleaned for next use and data analysed (C). The steps listed under each drawing refer to the ones described in the 655 experimental procedure.

\section{Figure 3: Custom devices for sleep detection and interference}

A. Snapshot of pySolo Video in action. Nocturnal view of flies in an arena, as seen by the webcam. Animals are in total darkness and infrared illumination comes from underneath the arena through infrared LEDs. Some of the grooves have an active recording mask delimiting the area where flies move (white rectangles). Each mask shows a midline point that is used by the program to emulate 
the beam-split system (vertical red line). Moving flies are highlighted by pySolo Video with a red rectangle, whose size is proportional to the extent of movement measured. A white cross on the fly indicates immobility. Rectangular masks can be drawn holding the left mouse button while dragging

665 the cursor on the image or can be drawn by clicking to indicate four corners to be connected for higher flexibility in drawing shapes (red crosses).

B-D. Three different ways of recording motion. Cartoons showing a fly walking through four different points in space. Motion of the animal can be recorded in three different formats. B. emulation of the infrared beam split. The position of the animal is calculated at every video frame

670 (about 15-25 times a second) but motion is recorded only if the fly is trespassing the transversal midline of the chamber (red line in panels B and A). In this example, motion would be recorded only when the fly moves from the lower point to the next. C. Measuring distance. The position of the fly is calculated at every frame and activity is measured by calculating the distance walked by the fly at each minute (blue line). D. The position of the fly is calculated at every frame, then saved

675 with resolution of one second. This latter format is to be considered as the raw data from which the other two can be derived. Additional custom analysis may provide also information on the activity of the animal, including the position held at any single time, speed and directions.

E. Schematics of how sleep is estimated and processed. Locomotor activity of the fly is plotted as function of time (leftmost, grey rectangles): each rectangle represents the distance walked by the fly

680 in one minute. To estimate sleep, activity data are first flattened to a binary matrix in which flies can be only inactive (black) or active (white) (middle bar); episodes of inactivity that last for at least five consecutive minutes are considered sleep (rightmost bar). In this example, the fly experiences three sleep bouts (black arrows) and two episodes of simple inactivity (blue arrow).

F. Real activity vs. virtual split-beam. Activity (upper panel) and sleep (lower panel) of a single fly

685 recorded for 24 hours and plotted calculating the actual distance of the flies as shown in panel c (light blue) and the virtual beam-split as explained in panel b (pink). Both methods show clearly the daily peaks of activity (two, in this case) which would be used for circadian analysis. However, only by analysing distance can the real activity and sleep be properly estimated and beam-split can overestimate sleep amount by more than $100 \%$ (see also reference 13 ).

690

\section{Figure 4: Components of Drosophila sleep}

A. A hypnogram showing how locomotor activity calculated with the virtual beam split method (upper panel) translates into sleep (lower panel).

The graph shows activity and sleep data for actual male (blue) and female (green) flies over a 24

695 hour period. Notice the difference in sleep during mid-day (the male siesta).

B. Graphic description of fly sleep over a 24 hour period in a 12:12 light/dark cycle. Each black box 
represents a single sleep bout. Wild-type flies sleep primarily in the night. The fly labelled 'disrupted' has shortened bout lengths, with a similar total sleep time to wild-type flies. This phenotype can be distinguished from long and short sleeping flies that have differences in total amount of sleep.

\section{Supplementary Figure 1: Photographs of the equipment.}
A. The recording monitor.
B. A printed arena. The transparent lid is secured using a rubber band. The middle chamber is filled 705 with food.

C. A3D printed arena compared to a glass tube traditionally used for infrared beam-split analysis. The size of the walking chamber is almost identical.

\section{Supplementary Video 1: Installing pySolo and pySolo video repositories in Ubuntu Linux}

710 A brief screencast guiding through the steps necessary to install the pySolo suite in Ubuntu linux adding custom repositories. The repositories will take automatic care of future updates.

\section{Supplementary Video 2: How to build a recording monitor}

This video shows what are the the basic components of a recording monitor and what are the

715 criteria to follow to build one of your own

\section{Supplementary Video 3: Preparing the arena for recording}

How to prepare an arena for recording: from transferring the food to placing the flies.

\section{Supplementary Video 4: Configuring the datafetcher script}

Configuring the datafetcher for automatic fetching and formatting of data. Can be used for pySolo Video or for the original TriKinetics Software.

\section{Supplementary Video 5: An introduction to pySolo Video}

725 A brief introduction showing how to configure and use pySolo Video

\section{Supplementary Video 6: Using pySolo for data analysis}

A detailed guide with complete instruction on how to use pySolo for data analysis. 
Supplementary Table 2: Comparing costs of video tracking and infrared beam-split 735

\section{Supplementary Data}

The zip file contains an electronic drawing of a complete arena, as shown in Supplementary figure 1b-c. The file, provided here in STL format, was created using the free software Blender

(http://www.blender.org) and can be opened and viewed using also the free STL viewer meshlab 740 (http://meshlab.sourceforge.net) 
Table 1 | Main differences between video tracking and split IR beam system

\begin{tabular}{|c|c|c|c|}
\hline & \multicolumn{2}{|c|}{ Split IR beam } & \multirow[t]{2}{*}{ Video tracking } \\
\hline & Single beam & Multiple beams & \\
\hline Spatial resolution & Low & medium to high & High \\
\hline $\begin{array}{l}\text { Movement } \\
\text { resolution }\end{array}$ & $\begin{array}{c}\text { Binary (crossed / did } \\
\text { not cross) }\end{array}$ & Up to $3 \mathrm{~mm}$ & $\begin{array}{l}\text { Can detect up to sub- } \\
\text { millimetrical movements }\end{array}$ \\
\hline $\begin{array}{l}\text { Interactions } \\
\text { among flies }\end{array}$ & Flies must be isolated & Flies must be isolated & $\begin{array}{c}\text { Flies can be interacting, with } \\
\text { caveats }\end{array}$ \\
\hline Scalability & Limited by cost & Limited by cost & High if using webcams \\
\hline Scalability (time) & $\begin{array}{c}\text { Limited by tube } \\
\text { preparation }\end{array}$ & $\begin{array}{l}\text { Limited by tube } \\
\text { preparation }\end{array}$ & High if using custom arena \\
\hline $\begin{array}{l}\text { Number of } \\
\text { monitors per } \\
\text { computer }\end{array}$ & Up to 120 & Up to 120 & Between 30 and $120[*]$ \\
\hline $\begin{array}{l}\text { Cost of a single } \\
\text { monitor }\end{array}$ & $\$ 500-\$ 800$ & $\$ 800-\$ 3200$ & $\$ 10-\$ 50$ \\
\hline $\begin{array}{c}\text { Promptness of } \\
\text { usage }\end{array}$ & Ready to use on arrival & Ready to use on arrival & $\begin{array}{l}\text { Need custom building, } \\
\text { testing }\end{array}$ \\
\hline $\begin{array}{l}\text { Adoption in the } \\
\text { field }\end{array}$ & $\begin{array}{l}\text { Popular with circadian } \\
\text { and sleep researchers }\end{array}$ & New & New \\
\hline
\end{tabular}


[*]. Technical specifications of the USB protocol allow for up to 127 devices to be connected to a 745 single computer. However, we have not yet tested more than 30 devices connected to the same machine and we take 30 as a "safe limit" in this protocol, also when estimating total costs. 
Table 2 | Comparing methods of sleep modulation

\begin{tabular}{ccccc}
\hline Method & $\begin{array}{c}\text { High - } \\
\text { throughput }\end{array}$ & $\begin{array}{c}\text { Specificity of } \\
\text { action }\end{array}$ & Controlled & Strength \\
\hline $\begin{array}{c}\text { Mechanical } \\
\text { (manual) }\end{array}$ & -- & +++ & +++ & $\begin{array}{c}\text { Will allow to target specifically } \\
\text { only flies that are asleep and leave } \\
\text { awake flies undisturbed }\end{array}$ \\
$\begin{array}{c}\text { Mechanical } \\
\text { (automatic) }\end{array}$ & +++ & ++ & - & $\begin{array}{c}\text { Highly scalable and quite specific } \\
\text { Pharmacological }\end{array}$ \\
& +++ & - & - & $\begin{array}{c}\text { Useful to test whether a gene is } \\
\text { involved with sleep regulation } \\
\text { through a given pathway }\end{array}$ \\
Social & + & $?$ & - & $\begin{array}{c}\text { Useful to test links between sleep } \\
\text { and social learning }\end{array}$ \\
$\begin{array}{c}\text { Starvation- } \\
\text { induced }\end{array}$ & +++ & $?$ & - & $\begin{array}{c}\text { Useful to test physiological } \\
\text { regulation of sleep wake cycle }\end{array}$ \\
\hline
\end{tabular}


Table 3 | Drugs used to manipulate sleep

\begin{tabular}{llll}
\hline Compound & Approximate effective dose & Reported effect & Reference \\
\hline Methamphetamine & $0.6-1.25 \mathrm{mg} / \mathrm{ml}$ & $\mathrm{W}$ & 27 \\
Octopamine & $5-10 \mathrm{mg} / \mathrm{ml}$ & $\mathrm{W}$ & 23 \\
Caffeine & $0.2-5 \mathrm{mg} / \mathrm{ml}$ & $\mathrm{W}$ & $3,4,28,29$ \\
CPT & $2.5 \mathrm{mg} / \mathrm{ml}$ & $\mathrm{W}$ & 28 \\
DPMX & $2.5 \mathrm{mg} / \mathrm{ml}$ & $\mathrm{W}$ & 28 \\
Cocaine & $0.5 \mathrm{mg} / \mathrm{ml}$ & $\mathrm{W}$ & 30 \\
Modafinil & $0.3-2.5 \mathrm{mg} / \mathrm{ml}$ & $\mathrm{W}$ & 31 \\
Cyclohexyladenosine & $0.5 \mathrm{mg} / \mathrm{ml}$ & $\mathrm{S}$ & 3 \\
Carbamazepine & $0.2-1.2 \mathrm{mg} / \mathrm{ml}$ & $\mathrm{S}$ & 32 \\
Hydroxyzine & $2.5-200 \mathrm{mg} / \mathrm{ml}$ & $\mathrm{S}$ & 4 \\
5-hydroxytryptophan & $1 \mathrm{mg} / \mathrm{ml}$ & $\mathrm{S}$ & 33 \\
3-iodo-tyrosine & $5 \mathrm{mg} / \mathrm{ml}$ & $\mathrm{S}$ & 26 \\
\hline
\end{tabular}

750

CPT: 8-Cyclopentyll-1,3-dimethlxanthin

DPMX: 3,7-Dimethyl-1-2-propynylxanthine

W: Increases wake time

$\mathrm{S}$ : Increases sleep time. 
Table 4 | Troubleshooting table.

\begin{tabular}{|c|c|c|c|}
\hline Step & Problem & Possible reason & Solution \\
\hline 7 & $\begin{array}{l}\text { Flies die or suffer after } \\
\text { sedation }\end{array}$ & $\begin{array}{l}\text { Flies are left too long on the } \\
\text { narcotic pad }\end{array}$ & $\begin{array}{l}\text { Be quicker when } \\
\text { sorting flies or use } \\
\text { pulses of } \mathrm{CO}_{2} \text { rather } \\
\text { than continuous flow. }\end{array}$ \\
\hline $8 \mathrm{~A}$ & $\begin{array}{l}\text { Flies die during transfer using } \\
\mathrm{CO}_{2} \text { anaesthesia }\end{array}$ & $\begin{array}{l}\text { Flies touch the food while still } \\
\text { unconscious and get stuck }\end{array}$ & $\begin{array}{l}\text { Pay attention not to } \\
\text { push flies against the } \\
\text { food }\end{array}$ \\
\hline \multirow[t]{2}{*}{$8 \mathrm{~B}$} & $\begin{array}{l}\text { Flies escape when transferring } \\
\text { them to the arena using mouth } \\
\text { pipette }\end{array}$ & Too many flies in the same vial & $\begin{array}{l}\text { Use vials with fewer } \\
\text { flies }\end{array}$ \\
\hline & & $\begin{array}{l}\text { Vials are closed with hard } \\
\text { pressed paper stoppers }\end{array}$ & $\begin{array}{l}\text { Replace stoppers with } \\
\text { cotton wool }\end{array}$ \\
\hline $8 B$ & $\begin{array}{l}\text { Flies die during transfer using } \\
\text { mouth pipette }\end{array}$ & $\begin{array}{l}\text { Inlet hole of the mouth pipette is } \\
\text { too small }\end{array}$ & $\begin{array}{l}\text { Increase diameter of } \\
\text { the hole }\end{array}$ \\
\hline 8 & $\begin{array}{l}\text { Flies escape from the recording } \\
\text { arena }\end{array}$ & Lid is not properly closed & $\begin{array}{l}\text { Use a rubber band or } \\
\text { scotch tape to close } \\
\text { properly the lid and } \\
\text { make it adhere to the } \\
\text { arena }\end{array}$ \\
\hline \multirow[t]{3}{*}{10} & $\begin{array}{l}\text { Program crashes or does not } \\
\text { start }\end{array}$ & $\begin{array}{l}\text { Version of the operating system } \\
\text { may not be supported }\end{array}$ & $\begin{array}{l}\text { Try installing a } \\
\text { supported and tested } \\
\text { OS, such as Linux } \\
\text { Ubuntu } 10.10 \text { or } \\
\text { Microsoft Windows } 7\end{array}$ \\
\hline & $\begin{array}{l}\text { Program crashes when playing } \\
\text { preview }\end{array}$ & $\begin{array}{l}\text { Resolution not supported by the } \\
\text { camera }\end{array}$ & $\begin{array}{l}\text { Try setting camera } \\
\text { resolution to } 640 \times 480\end{array}$ \\
\hline & $\begin{array}{l}\text { Program crashes when using } \\
\text { multiple cameras }\end{array}$ & $\begin{array}{l}\text { Cameras are drawing too much } \\
\text { current }\end{array}$ & $\begin{array}{l}\text { Connect cameras to an } \\
\text { USB hub with an } \\
\text { independent power } \\
\text { supplier }\end{array}$ \\
\hline 15 & Image is blurry & Camera objective is out of focus & $\begin{array}{l}\text { Adjust focus manually } \\
\text { and, if necessary, apply } \\
\text { some glue to lock the } \\
\text { lens in place }\end{array}$ \\
\hline 28 & $\begin{array}{l}\text { Flies die during sleep } \\
\text { manipulation }\end{array}$ & $\begin{array}{l}\text { Sleep deprivation lasting too } \\
\text { long }\end{array}$ & $\begin{array}{l}\text { Reduce the duration of } \\
\text { sleep deprivation }\end{array}$ \\
\hline \multirow[t]{3}{*}{$28 \mathrm{~B}$} & $\begin{array}{l}\text { Flies die during sleep } \\
\text { manipulation }\end{array}$ & Concentration of drug is too high & $\begin{array}{l}\text { Reduce the } \\
\text { concentration of drug }\end{array}$ \\
\hline & Flies do not eat the drug & $\begin{array}{l}\text { Drug tastes unpleasant and flies } \\
\text { will not eat it }\end{array}$ & $\begin{array}{l}\text { Try changing the food } \\
\text { mixture }\end{array}$ \\
\hline & $\begin{array}{l}\text { Sleep pattern is not very } \\
\text { different between day and night }\end{array}$ & Too much light during the night & $\begin{array}{l}\text { Make sure the } \\
\text { incubator is properly }\end{array}$ \\
\hline
\end{tabular}


sealed or, if necessary, use blackout foil to close residual openings.

Not enough light during the day Make sure the lights are properly connected and will turn on during the day

Flies die after few days of $\quad$ Food dries out and flies have recording
Make sure that humidity in the incubator is at desired levels

Use colder water or use a different material for printing 


\section{Box 1 | GLOSSARY}

Arousal stimulus: a sensory stimulus that will induce motion in an otherwise immobile fly. Can be

a mechanical stimulus such as a vibration induced by tapping a finger against the recording chamber.

Arousal threshold: the threshold beyond which a certain stimulus will promote motion. When the fly is asleep the arousal threshold is higher than when it is awake but immobile. Also, longer periods of inactivity correlate with even higher arousal threshold suggesting that Drosophila sleep can also be divided into light sleep and deep sleep ${ }^{16}$.

Sleep episode or bout: an episode of continuous immobility lasting at least five consecutive minutes. Its duration is measured in minutes.

Sleep latency: time passing between light off and the start of the first sleep episode at night. It is used as a read out of the mechanisms underlying sleep initiation.

770 Sleep cycle: Interval from the beginning of one sleep episode to the beginning of the subsequent sleep episode.

Sleep fragmentation: indicates the number of sleep episodes in a sleep cycle

Sleep duration: the cumulative amount of sleep in a 24 hours period measured in minutes.

Baseline sleep: the sleeping pattern in undisturbed conditions

775 Sleep deprivation: the act of forcefully keeping the fly awake

SNAP: Sleep nullifying apparatus. A machine that sleep deprives flies by rotating on one axis and interfering with their geotactic sense

Sleep loss: the amount of sleep lost upon sleep deprivation, measured in minutes and calculated against baseline sleep

780 Recovery sleep or sleep rebound: the amount of sleep recovered at the end of sleep deprivation. It is frequently calculated looking at sleep during the $6,12,24$ or $48 \mathrm{~h}$ period following the end of sleep deprivation, using baseline sleep as reference value.

Activity count: an absolute measure of the locomotor activity of the flies. When activity is measured using the infrared beam-split system, the activity count equals the number of beam

785 crosses per time unit. When activity is measured using video analysis, activity count equals the distance per time unit.

Activity index (AI): a relative measure of locomotor health. AI is calculated dividing the activity count by the total time spent awake in a 24 hours period. This is a way to normalize activity among flies with different total sleep duration. 


\section{References}

1. Roberts, S.K. "Clock" Controlled Activity Rhythms in the Fruit Fly. Science 124, 172(1956).

2. Campbell, S.S. \& Tobler, I. Animal sleep: a review of sleep duration across phylogeny. Neurosci Biobehav Rev 8, 269-300(1984).

3. Hendricks, J.C., Finn, S.M., Panckeri, K.A., Chavkin, J., Williams, J.A., Sehgal, A. et al. Rest in Drosophila is a sleep-like state. Neuron 25, 129-38(2000).

4. Shaw, P.J., Cirelli, C., Greenspan, R.J. \& Tononi, G. Correlates of sleep and waking in Drosophila melanogaster. Science 287, 1834-7(2000).

5. Graves, L.A., Heller, E.A., Pack, A.I. \& Abel, T. Sleep deprivation selectively impairs memory consolidation for contextual fear conditioning. Learn. Mem. 10, 168-76(2003).

6. Li, X., Yu, F. \& Guo, A. Sleep deprivation specifically impairs short-term olfactory memory in Drosophila. Sleep 32, 1417-24(2009).

7. Gilestro, G.F., Tononi, G. \& Cirelli, C. Widespread changes in synaptic markers as a function of sleep and wakefulness in Drosophila. Science 324, 109-12(2009).

8. Ganguly-Fitzgerald, I., Donlea, J. \& Shaw, P.J. Waking experience affects sleep need in Drosophila. Science 313, 1775-81(2006).

9. Nitz, D.A., van Swinderen, B., Tononi, G. \& Greenspan, R.J. Electrophysiological correlates of rest and activity in Drosophila melanogaster. Curr. Biol. 12, 1934-40(2002).

10. Pfeiffenberger C, Lear BC, Keegan KP, Allada R. Locomotor Activity Level Monitoring Using the Drosophila Activity Monitoring (DAM) System. Cold Spring Harb Protoc. 11, 2010 11. Konopka, R.J. \& Benzer, S. Clock mutants of Drosophila melanogaster. Proc. Natl. Acad. Sci. U.S.A. 68, 2112-6(1971).

12. Rosato, E. \& Kyriacou, C.P. Analysis of locomotor activity rhythms in Drosophila. Nat Protoc 1, 559-68(2006).

13. Zimmerman, J.E., Raizen, D.M., Maycock, M.H., Maislin, G. \& Pack, A.I. A video method to study Drosophila sleep. Sleep 31, 1587-98(2008).

14. Gilestro, G.F. \& Cirelli, C. pySolo: a complete suite for sleep analysis in Drosophila. Bioinformatics 25, 1466-7(2009).

15. Chiu, J.C., Low, K.H., Pike, D.H., Yildirim, E. \& Edery, I. Assaying locomotor activity to study circadian rhythms and sleep parameters in Drosophila. J Vis Exp , (2010).

16. Huber, R., Hill SL, Holladay C, Biesiadecki M, Tononi G, Cirelli C. Sleep homeostasis in Drosophila melanogaster. Sleep 27,628-39(2004).

17. Keene, A.C., Duboué, E.R., McDonald, D.M., Dus, M., Suh, G.S.B., Waddell, S. et al. Clock and cycle limit starvation-induced sleep loss in Drosophila. Curr. Biol. 20, 1209-15(2010). 
18. Shaw, P.J., Tononi, G., Greenspan, R.J. \& Robinson, D.F. Stress response genes protect against lethal effects of sleep deprivation in Drosophila. Nature 417, 287-91(2002).

19. Shimizu H, Shimoda M, Yamaguchi T, Seong KH, Okamura T, Ishii S. Drosophila ATF-2 regulates sleep and locomotor activity in pacemaker neurons. Mol Cell Biol, 28, 6278-89(2008)

20. Branson, K., Robie, A.A., Bender, J., Perona, P. \& Dickinson, M.H. High-throughput ethomics in large groups of Drosophila. Nat. Methods 6, 451-7(2009).

21. Straw, A.D. \& Dickinson, M.H. Motmot, an open-source toolkit for realtime video acquisition and analysis. Source Code Biol Med 4, 5(2009).

22. Catterson, J.H., Knowles-Barley, S., James, K., Heck, M.M.S., Harmar, A.J. \& Hartley, P.S. Dietary modulation of Drosophila sleep-wake behaviour. PLoS ONE 5, e12062(2010).

23. Crocker, A. \& Sehgal, A. Octopamine regulates sleep in Drosophila through protein kinase Adependent mechanisms. J. Neurosci. 28, 9377-85(2008).

24. Hendricks, J.C., Lu, S., Kume, K., Yin, J.C.P., Yang, Z. \& Sehgal, A. Gender dimorphism in the role of cycle (BMAL1) in rest, rest regulation, and longevity in Drosophila melanogaster. J. Biol. Rhythms 18, 12-25(2003).

25. Koh, K., Evans, J.M., Hendricks, J.C. \& Sehgal, A. A Drosophila model for age-associated changes in sleep:wake cycles. Proc. Natl. Acad. Sci. U.S.A. 103, 13843-7(2006).

26. Andretic, R. \& Shaw, P.J. Essentials of sleep recordings in Drosophila: moving beyond sleep time. Meth. Enzymol. 393, 759-72(2005).

27. Andretic, R., van Swinderen, B. \& Greenspan, R.J. Dopaminergic modulation of arousal in Drosophila. Curr. Biol. 15, 1165-75(2005).

28. Andretic, R., Kim, Y., Jones, F.S., Han, K. \& Greenspan, R.J. Drosophila D1 dopamine receptor mediates caffeine-induced arousal. Proc. Natl. Acad. Sci. U.S.A. 105, 20392-7(2008).

29. Wu, M.N., Ho, K., Crocker, A., Yue, Z., Koh, K. \& Sehgal, A. The effects of caffeine on sleep in Drosophila require PKA activity, but not the adenosine receptor. J. Neurosci. 29, 1102937(2009).

30. Lebestky, T., Chang, J.C., Dankert, H., Zelnik, L., Kim, Y., Han, K. et al. Two different forms of arousal in Drosophila are oppositely regulated by the dopamine D1 receptor ortholog DopR via distinct neural circuits. Neuron 64, 522-36(2009).

31. Hendricks, J.C., Kirk, D., Panckeri, K., Miller, M.S. \& Pack, A.I. Modafinil maintains waking in the fruit fly drosophila melanogaster. Sleep 26, 139-46(2003).

32. Agosto, J., Choi, J.C., Parisky, K.M., Stilwell, G., Rosbash, M. \& Griffith, L.C. Modulation of GABAA receptor desensitization uncouples sleep onset and maintenance in Drosophila. Nat.

Neurosci. 11, 354-9(2008).

33. Yuan, Q., Joiner, W.J. \& Sehgal, A. A sleep-promoting role for the Drosophila serotonin 
receptor 1A. Curr. Biol. 16, 1051-62(2006). 
a

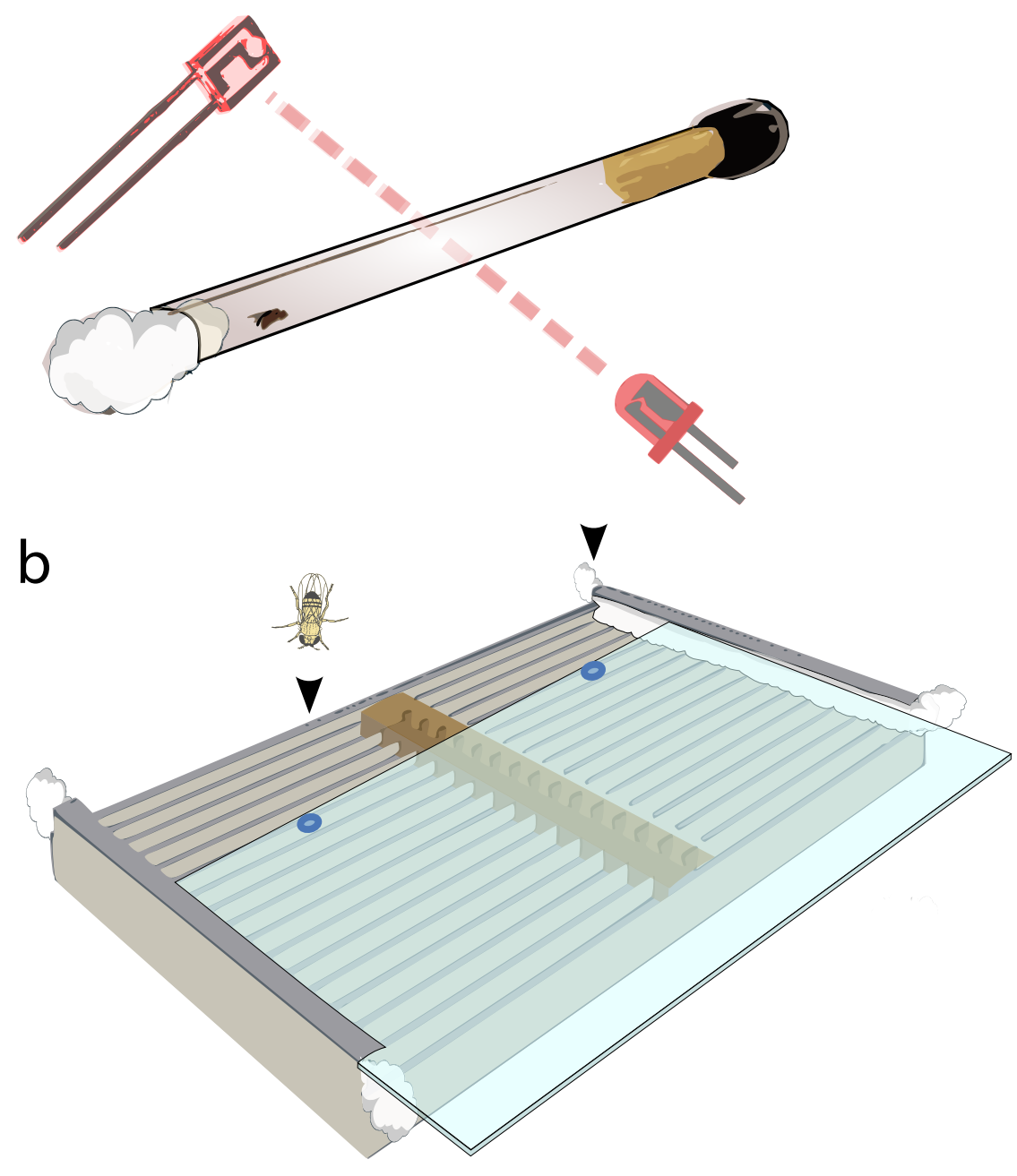

C

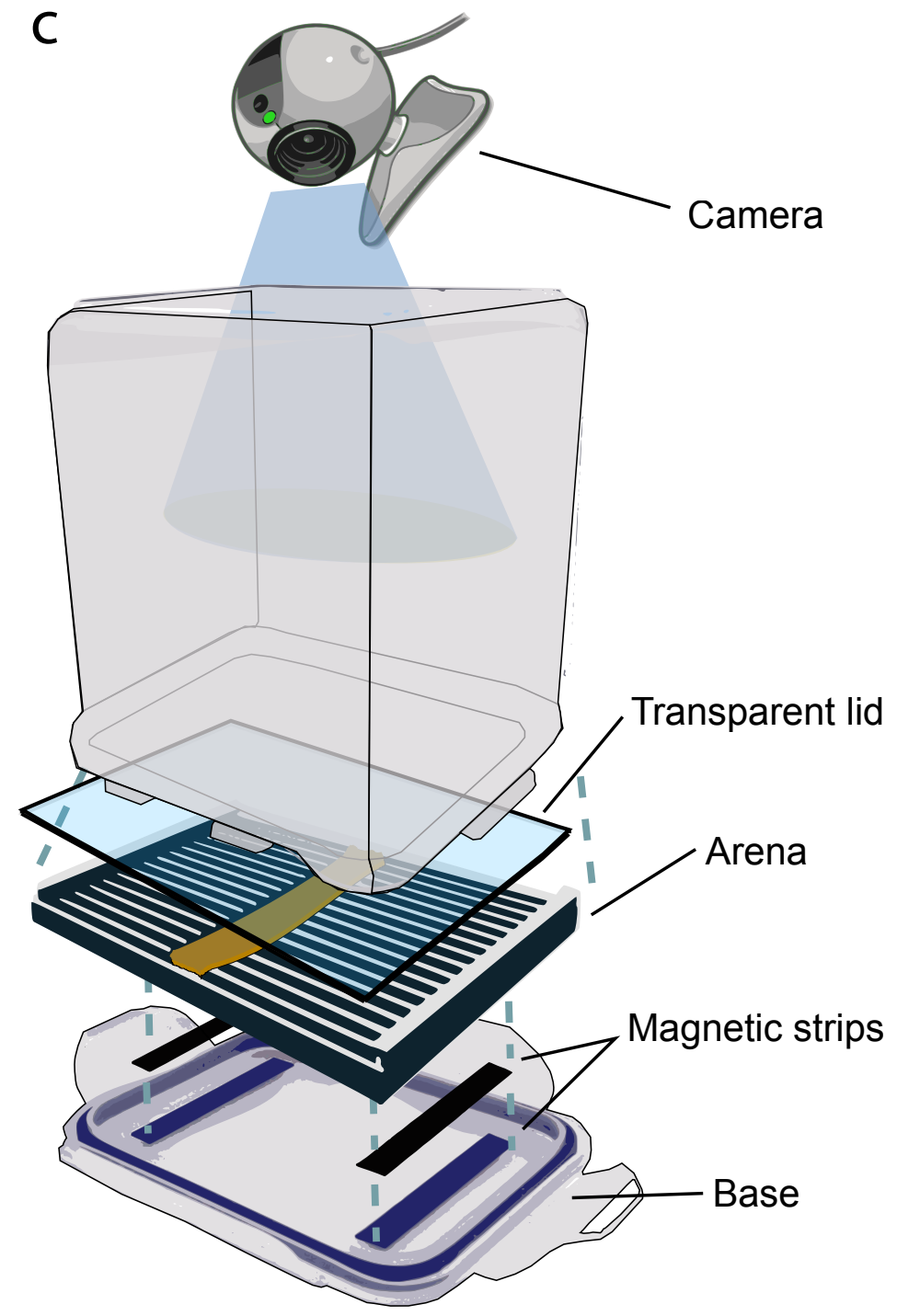


a

$$
\text { preparation of the arena }
$$

b

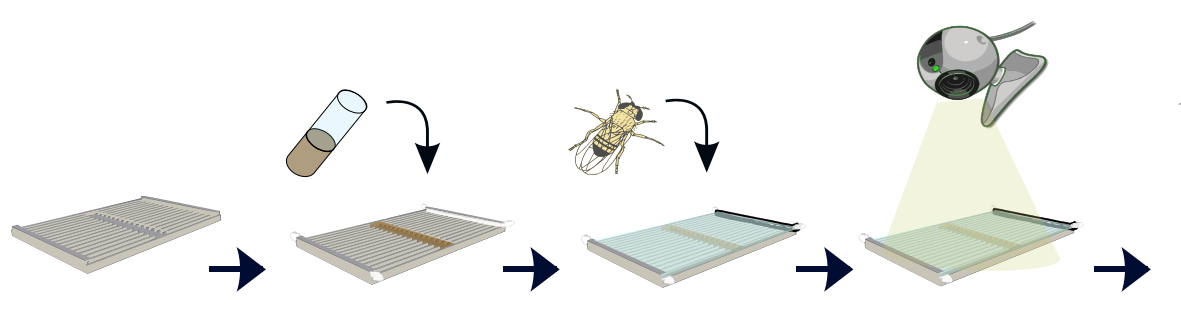

add food add flies

baseline sleep 2-3 days

steps 3-6

sleep analyis data analysis

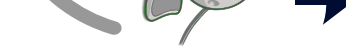

sleep deprivation 3-24 hours step 28

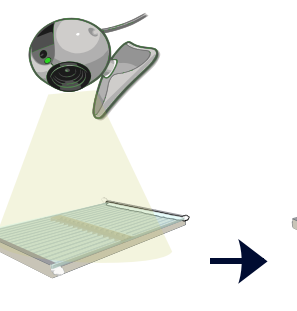

recovery 1-3 days step 29 clean arena

steps $30-33$

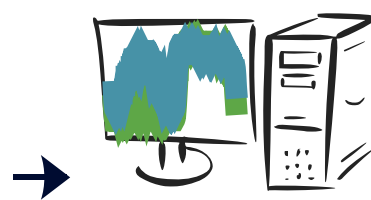

steps 34-49 


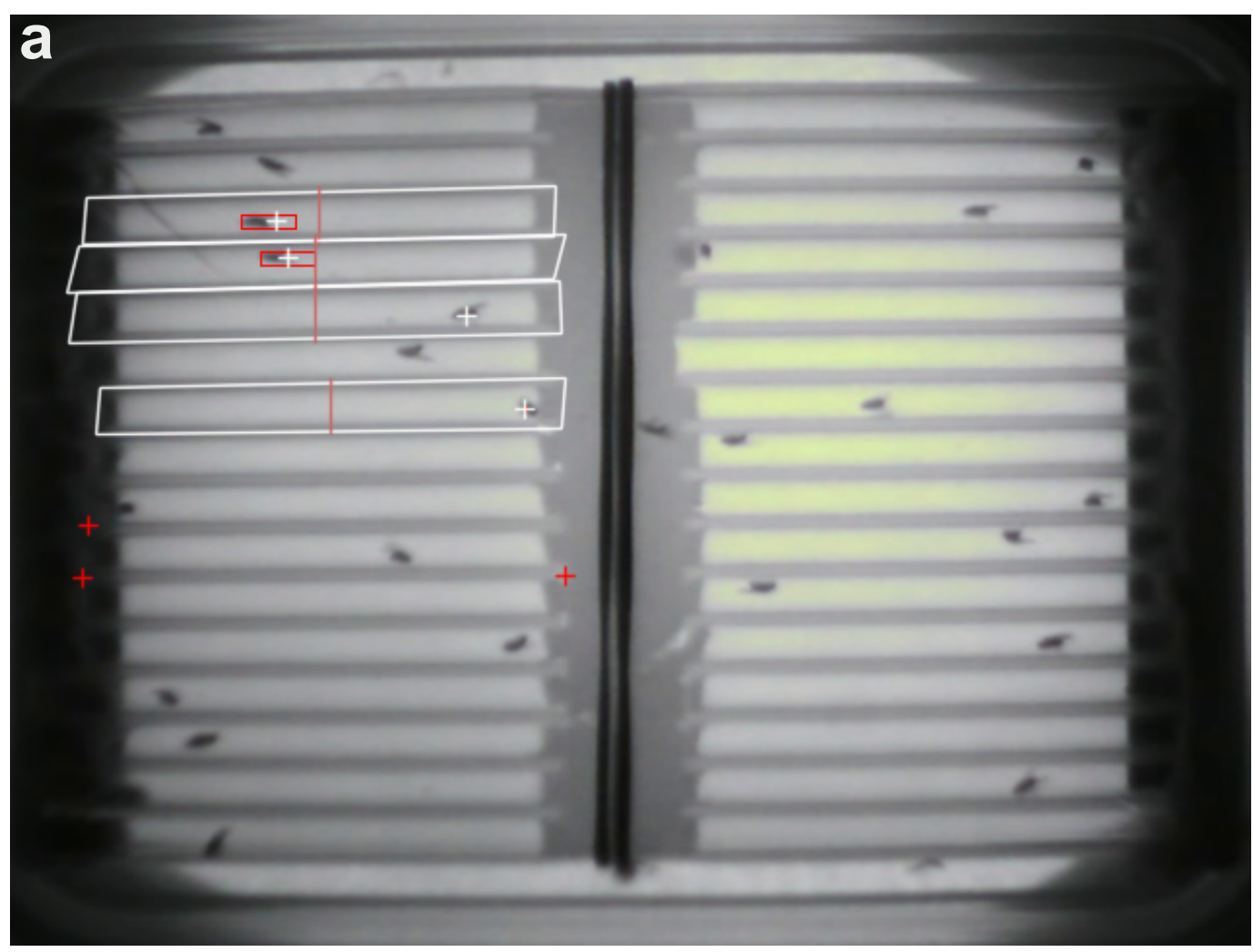

e activity

$\frac{0}{4}$ abs. bin. $\frac{\mathscr{Q}}{n}$

b

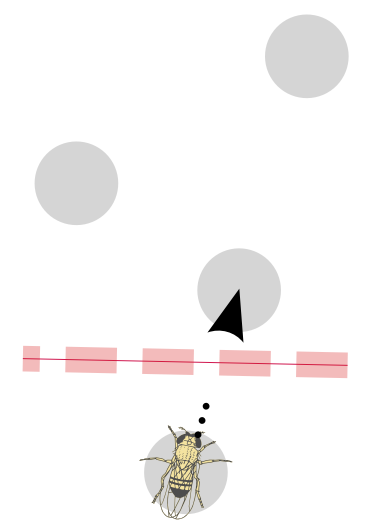

C

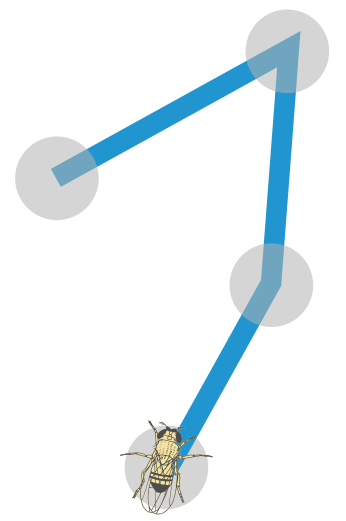

d

f $\quad$ virtual beam split

호ํ

5
4
3
2
1
0

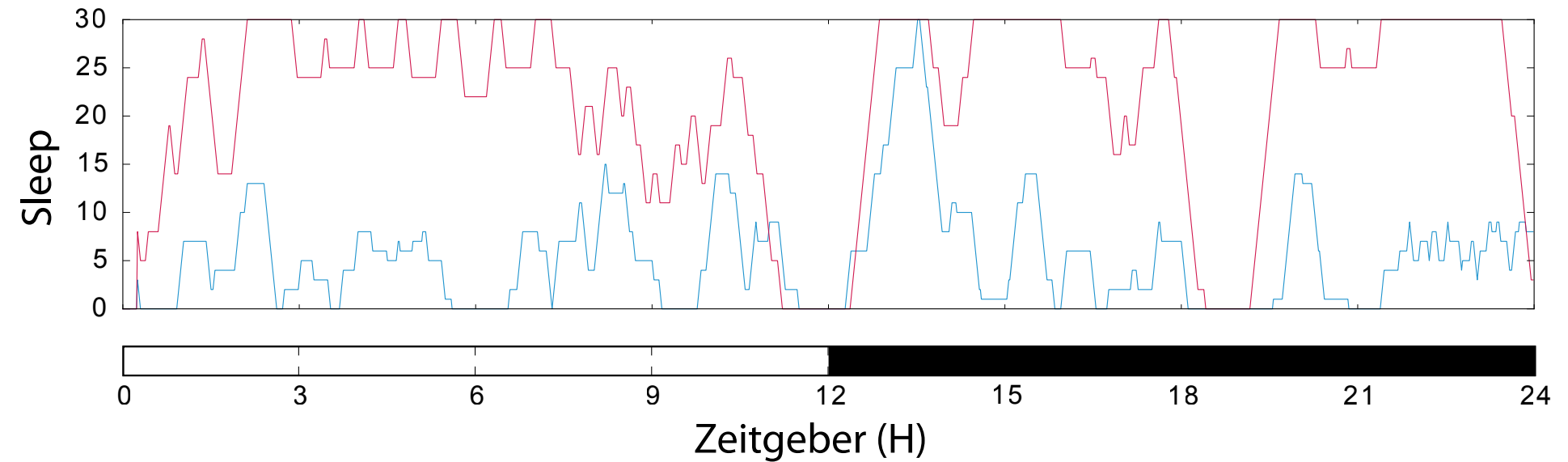




\section{a}
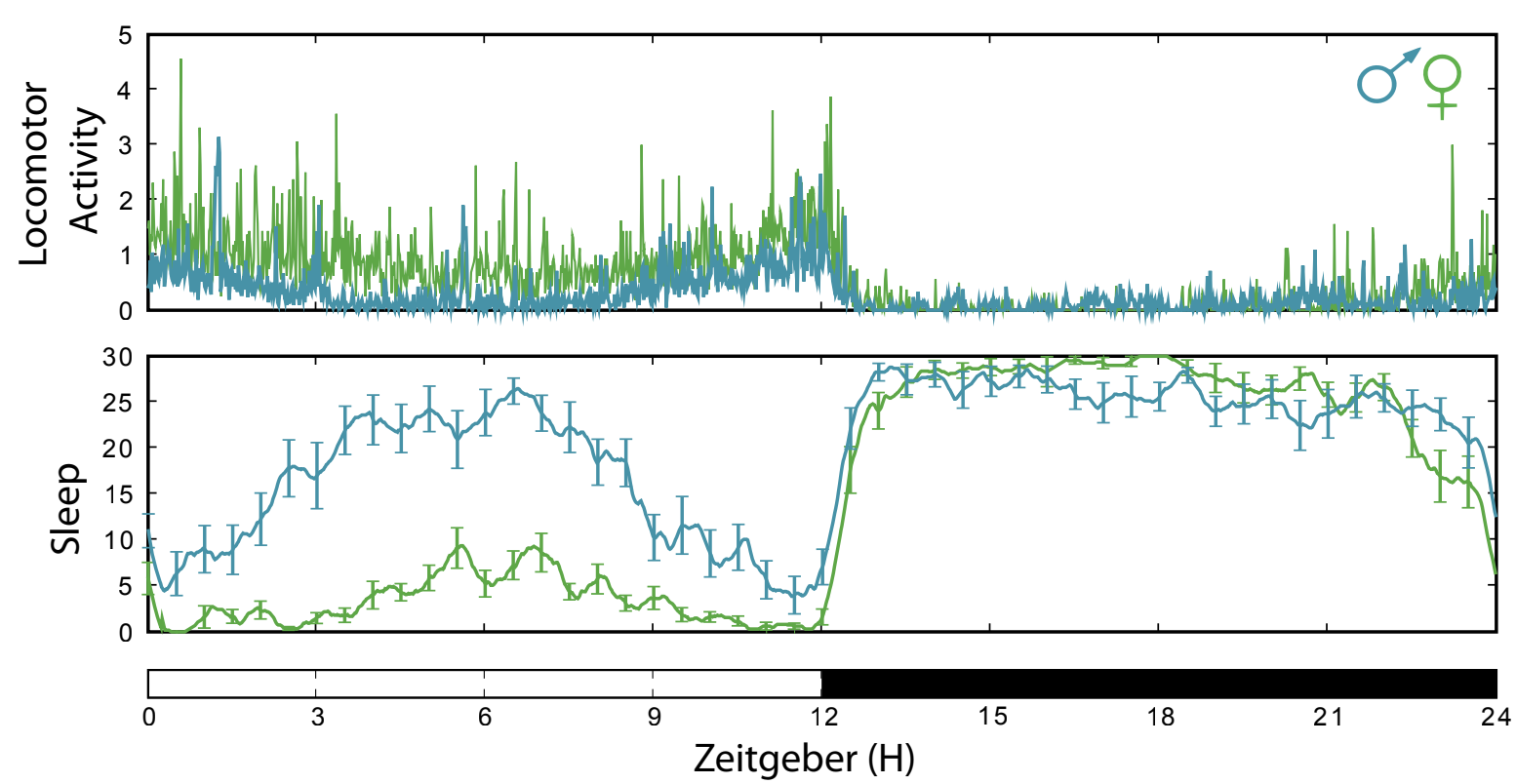

b

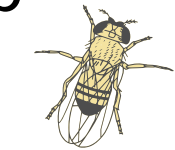

0

12

normal

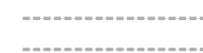

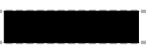

disrupted

WD

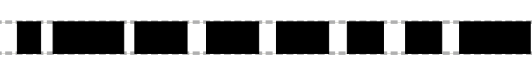

long

short

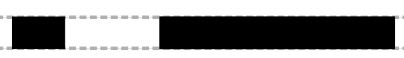




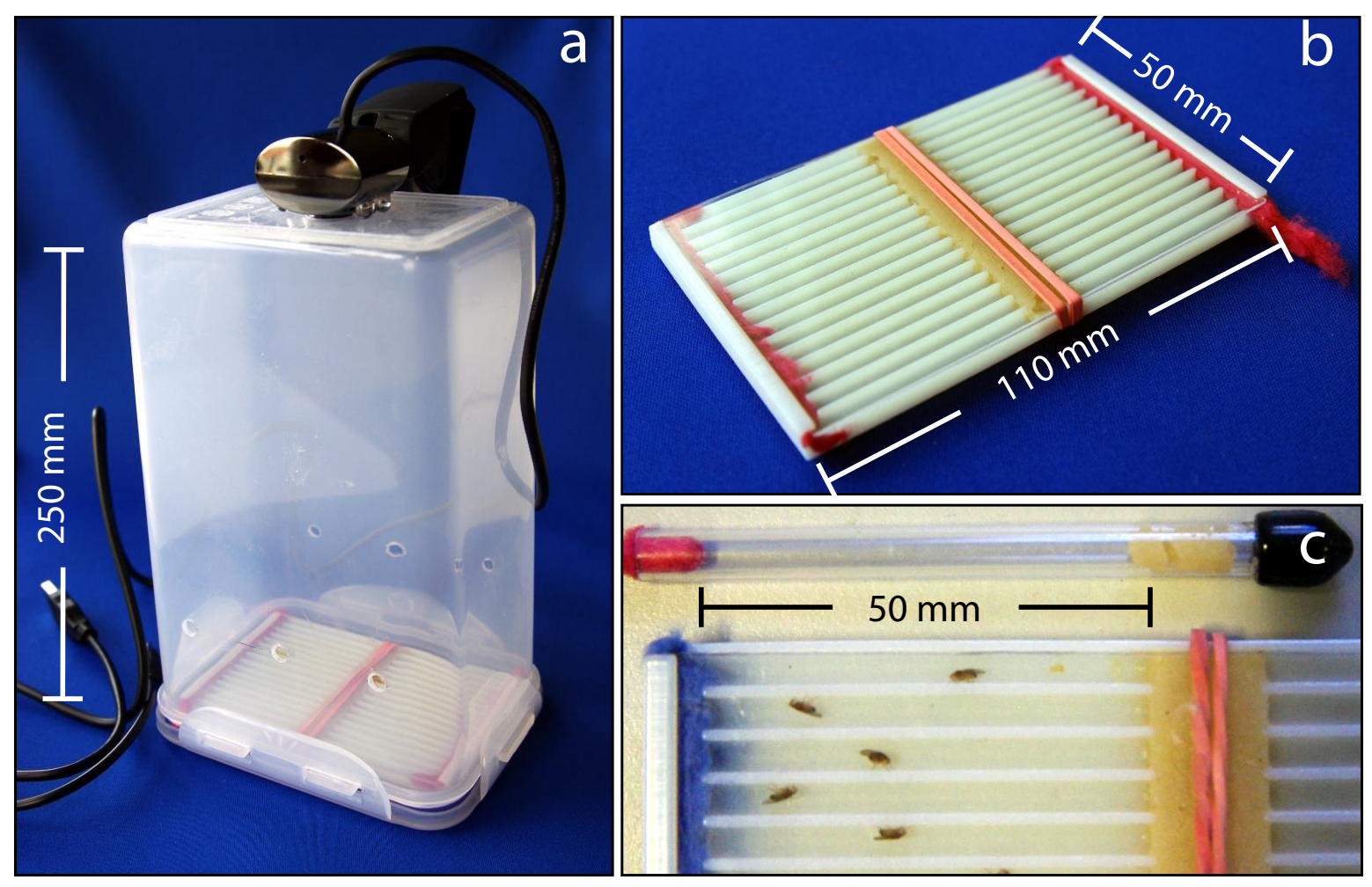

